\title{
Scrum Practices and Global Software Development
}

\author{
Areej Sewalh AL_Zaidi, M. Rizwan Jameel Qureshi \\ Faculty of Computing and Information Technology, King Abdulaziz University, Jeddah, 21589, Saudi Arabia \\ Email: d_ream_girl@hotmail.com,rmuhammd@kau.edu.sa
}

\begin{abstract}
Global software development (GSD) is one of the emerging areas of software engineering. Stakeholders are distributed located that brings into several challenges to coordinate and collaborate effectively. Scrum is one the widely practiced agile methodologies. Majority of the software development companies showed a great interest from last few years to apply Scrum methodology in distributed development to solve the coordination and communication problems. Although Scrum contains good features and strengths but still there are improvement possibilities to solve the problems of coordination and collaboration in GSD. Majority of software development companies face the problems of coordination and communication in GSD projects. This paper proposes a novel solution to mitigate the coordination and communication challenges of GSD projects. The survey is used as a research methodology to evaluate the proposed solution. The proposed solution is supported by $62.24 \%$. We anticipate that the proposed solution will help the software development companies to mitigate the coordination and communication challenges of GSD projects.
\end{abstract}

Index Terms-GSD, coordination, communication, Scrum, mitigation strategies.

\section{INTRODUCTION}

There is a shift in software industry from centralized to distributed development from last several years. The main benefits of this shift are time saving, cost saving, improve quality, round-the-clock development, access to cheap and skilled resources, and high productivity. On the other hand, global software development (GSD) suffers many challenges like effective coordination and collaboration among stakeholders at different geographical locations with different time zones. Poor coordination and communication may lead to fail GSD projects.

Agile models were introduced to complete the project with close interaction, collocated and face to face communication among the stakeholders. Agile models are introduced to manage and develop projects with tight and rigid deadlines. The main focus of these methodologies is to deliver software in iterations. Agile development needs strong teamwork, strong collaboration and process adaptability during whole development process [1]. Scrum is one of the widely practiced agile models. It is an incremental model that exhibits reliability, responsiveness and flexibility during the software development [2]. Scrum focuses more on project management as compared to other agile methodologies. The main goal, using Scrum, is to deliver working software frequently with regular time intervals.

Collaboration and coordination are closely related activities helping team members to work together to achieve common goals. The main feature of collaboration is to focus on problem solving aspects of team work. Coordination is a set of synchronized actions that team members take to achieve common goals. These two processes depend on one another and have similar requirements for shared understanding therefore collaboration and coordination come together in most of time to support each other. Coordination is going to play an important role in software development to successfully manage complex interdependencies among software tasks to improve performance of team members and meet timelines.

The need of high level collaboration and coordination among team members is urged to build quality software. Strong collaboration increase performance of team members. Coordination is one of important feature for teams to achieve common goals by sharing their interest and aligning different views. GSD is based on distributed sites and stakeholders require high coordination and communication. It is a great challenge for the software companies to achieve high coordination and communication in distributed development projects. This research mainly focuses on the coordination and communication problems of distributed teams. A novel solution is proposed to mitigate the coordination and communication problems of GSD projects.

The paper is further organized as follows. Related work is described in section II. Section III describes research question and depicts the details of proposed solution. Section IV illustrates the evaluation of proposed solution. Conclusion and future work is provided in section $\mathrm{V}$.

\section{RELATED WORK}

According to Ramanujam et al. [1], Scrum model is getting popularity from last few years due to its flexibility, responsiveness and reliability. Ramanujam et al. [1] propose a collaborative agile Scrum software framework to develop large scale systems. It is imperative that 
organizations engage multiple specilaized vendors to develop large systems like Microsoft Corp., Oracle Corp. and SAP AG.
Requirements Understanding (RU) is a severe challenge to software companies of Saudi Arabia those are involved in GSD [2]. The main challenges in GSD are

Table 1. Summary of the Related Work

\begin{tabular}{|c|c|}
\hline Paper Title with reference number & Summary \\
\hline $\begin{array}{l}\text { Requirements Understanding: A Challenge in Global Software } \\
\text { Development [1] }\end{array}$ & $\begin{array}{l}\text { Requirements understanding (RU) is a severe challenge for KSA software } \\
\text { companies that are involved in GSD. }\end{array}$ \\
\hline $\begin{array}{l}\text { Scrum Practice Mitigation of Global Software Development } \\
\text { Coordination Challenges: A Distinctive Advantage? [2] }\end{array}$ & $\begin{array}{l}\text { Scrum practices are used in four distributed GSD projects to show the } \\
\text { benefits of agile methods vs. traditional software engineering methods. }\end{array}$ \\
\hline $\begin{array}{l}\text { Problems We All Know We Have Them. Do We Have Solutions } \\
\text { Too [3] }\end{array}$ & $\begin{array}{l}\text { The main challenges in GSD are described and a solution is proposed to } \\
\text { solve these challenges. }\end{array}$ \\
\hline $\begin{array}{l}\text { Risk Identification and Mitigation Processes for Using Scrum in } \\
\text { Global Software Development: A Conceptual Framework [4] }\end{array}$ & $\begin{array}{l}\text { Illustrate of the key risks of GSD and current strategies to deal with these } \\
\text { risks. }\end{array}$ \\
\hline $\begin{array}{l}\text { Scrum Practices in Global Software Development: A Research } \\
\text { Framework [5] }\end{array}$ & $\begin{array}{l}\text { Project stakeholder distribution is described in GSD to characterize } \\
\text { temporal, geographical and socio-cultural challenges. }\end{array}$ \\
\hline $\begin{array}{l}\text { From RUP to Scrum in Global Software Development: A Case } \\
\text { Study [6] }\end{array}$ & A case study is conducted using Scrum in global software development. \\
\hline $\begin{array}{l}\text { Risk Identification and Risk Mitigation Instruments for Global } \\
\text { Software Development: Systematic Review and Survey Results [7] }\end{array}$ & $\begin{array}{l}\text { A survey is used to identify main challenges and their mitigation strategies } \\
\text { in GSD. }\end{array}$ \\
\hline $\begin{array}{l}\text { Collaborative and Competitive Strategies for Agile Scrum } \\
\text { Development [8] }\end{array}$ & $\begin{array}{l}\text { Propose a collaborative agile scrum software development framework for } \\
\text { complex multi-vendor competing environments. }\end{array}$ \\
\hline $\begin{array}{l}\text { On the Difficulties for Students to Adhere to Scrum on Global } \\
\text { Software Development Projects: Preliminary Results [9] }\end{array}$ & Scrum process is used in GSD. \\
\hline $\begin{array}{l}\text { Guiding Global Software Development Projects using Scrum and } \\
\text { Agile with Quality Assurance [10] }\end{array}$ & $\begin{array}{l}\text { Scrum is used to develop mobile applications for multiple mobile } \\
\text { platforms. }\end{array}$ \\
\hline Communication Issues in GSD [11] & $\begin{array}{l}\text { One of the main challenges of GSD practices is to ensure effective } \\
\text { communication among the team members }\end{array}$ \\
\hline $\begin{array}{l}\text { Global Software Development using Agile Methodologies: A } \\
\text { Review of Literature [12] }\end{array}$ & Systematic review of using agile methodologies like Scrum in GSD. \\
\hline $\begin{array}{l}\text { The agile requirements refinery applying SCRUM principles to } \\
\text { software [14] }\end{array}$ & $\begin{array}{l}\text { Although agile software development methods such as SCRUM is gaining } \\
\text { popularity, the consequences of applying agile principles to software } \\
\text { product management have received little attention until now. }\end{array}$ \\
\hline
\end{tabular}

communication problem, culture diversity, coordination, geographical distance and temporal difference. The culture diversity and communication are the most challenging issues. One of the main challenges of GSD is to ensure effective coordination and comunnication among the team members[3]. Shah et al. identified an ontology based solution to facilitate communication, modularization of work, study cognitive nature and characteristics of people and training on cultural norms. Shah et al. [4] describe that the agile methodologies are best suitable to develop GSD projects. Sriram [5] reported in support of using agile methodologies in GSD that the principles of Scrum and global software development are apparently contradictory in many areas but even then Scrum is one of the widely used methodologies in GSD due to its felxibility and good practices.

Hossain et al. [6] proposed a conceptual framework for GSD projects and key risks in GSD projects were also identified. A GSD project was reported in which students were distributed across three countries to develop mobile solutions using Scrum model and IBM Rational Team Concert tool [7]. The teams faced many difficulties such as poor knowledge about roles of developer, product owner and Scrum master, retrospective, sprint planning and burn down chart [7]. Scharff [8] proposed an extended Scrum model for GSD projects. The proposed model was validated using a GSD project to develop mobile applications [8].

Hang [9] describes a GSD project adopt Scrum for the development of an educational mobile application. Hang [9] concluds some important management principles. Vanessa and Sabrina [10] describe about GSD that the challenges intruded by geographical, temporal, and cultural distance are numerous. A systematic review is conducted on GSD literature and performed a survey to gather challenges and their mitigation strategies [11]. The main categories are communication, coordination, and control based on geographical distance, temporal distance and socio-culture distance. Forty eight challenges and forty two mitigation strategies are reported as a result of the survey. It is concluded that all the challenges and strategies are prevalent in certain industrial settings [11].

A transition from RUP to Scrum brings a positive effect in requirement's engineering, communication, cost management and cross-functionality of the distributed teams [12]. In this way, agile practices can have a positive impact on GSD projects and can help to mitigate many of the well-known challenges of GSD [12].

The distribution of teams in GSD project is characterized by temporal, geographical and sociocultural distance and it causes challenges of communication, coordination and control [13]. Practitioners are constantly seeking strategies, practices 
and tools to counter the challenges of GSD 13]. It is examined that Scrum methodology provide any distinctive advantage in mitigating coordination challenges as compared to the traditional methodologies [14]. It was validated by applying Scrum on four GSD case studies. It was found (as a result of the case studies) that scrum offers distinctive advantages in mitigating geographical and socio-cultural challenges of GSD projects. Scrum was not effective to control the temporal distance-based GSD coordination challenge [14].

Qureshi and Alsulami [15] focus on how to use Scrum to offer a distinctive feature in mitigating the effect of increased coordination costs in GSD projects. This is accomplished by offering a solution to divide the Scrum meetings into local meeting and global meeting to reduce the time of communication between distributed teams. In addition, develop (Distributed Scrum Web Application) to facilitate the communication among distributed team members and provide various services. So, the proposed solution offers a distinctive feature to mitigate the effects of "increased coordination costs" challenge on coordination in GSD projects.

There are many articles present on how Scrum methodology is implemented in GSD environment. The experience of a global company that implemented Scrum in its two projects is mentioned in [16]. It maintained its centers in Americas and Asia. Both projects experienced challenges when Scrum was implemented. One of the challenges was in communication as the teams did not attend remote Scrum meetings and that affected collaboration between them. A bigger challenge was in changing the culture of the company from waterfall to Scrum because there was some resistance by people within the company.

The biggest challenge for implementing Scrum in GSD environment is lack of synchronous communication as reported in [17]. This paper also defined the challenging factors of using Scrum practices in GSD projects and strategies to reduce these factors. While the authors of [18] suggest a framework of research which shows how practices of Scrum can mitigate challenges in GSD. It also defines three categories of challenges in GSD that are control, communication and coordination according to socio-cultural, geographical and temporal distance. However, this framework needs to validate empirically because it is a theoretical proposition.

An empirical study to implement Scrum practices in GSD projects is reported in [19]. This study was conducted to show the distinctive benefits of applying Scrum method in GSD projects over the traditional methods to mitigate GSD coordination challenges. The result was that Scrum method offered a distinctive feature to mitigate the effects of socio-cultural and geographical, but not temporal distance on coordination in GSD projects. There is a new method for GSD like scRumUP is mentioned in [20]. It integrated the advantages of Rational Unified Process (RUP) that is used for developing software and Scrum that is performed for managing a project. Table 1 illustrates the summary of related work.

\section{THE RESEARCH QUESTION AND THE PROPOSED SOLUTION}

Scrum model is getting popularity from last few years due to following reasons. Scrum model works well for small and medium projects and it can also be scaled for large projects. Scrum teams typically work in iterations (called sprints) that are from two weeks to one month long. Scrum teams do not allow changes into their sprints. Once the sprint planning meeting is completed and a commitment made to deliver a set of product backlog items, that set of items remains unchanged through the end of the sprint. Scrum product owner prioritizes the product backlog but the team decides the sequence in which they will develop the backlog items. Scrum is good in management but lacks in engineering practices.

The research question is:

Does Scrum mitigate geographical distance coordination and communication challenges in GSD?

Agile models allow face to face communication. Face to face communication is an efficient mechanism to share information for collocated teams but it does not work for distributed teams. Geographical distance is a measure of the effort required for travelling between sites and it very necessary for the relationships (team spirit) between the distributed teams. Limited face-to-face meetings can reduce possibility of informal contact by reducing team awareness and causing lack of team cohesiveness, interaction and management. There is a pressing need of suitable mechanisms to mitigate geographical distancebased communication challenges to get the benefits of Scrum in GSD. Following are the proposed mitigation strategies to handle coordination and communication challenges in GSD.

\section{A. Multiple Communication Modes}

Distributed teams must select a suitable communication tool like TeamViewer.

- Synchronous communication tools: It allows people to use the same time and different place mode. It enables real-time communication among teams. Drawbacks of synchronous tools are cost and high communication bandwidth. The most common synchronous tools are audio conferencing, web conferencing, video conferencing, chat, instant messaging, telephone, net meeting and shared mailing list.

- Asynchronous communication tools: It allows people to use the different time-different place communication mode. The most common asynchronous tools are discussion boards, web logs (blogs), messaging (e-mail), streaming audio, streaming video and desktop and application sharing.

\section{B. Version One's Project Management Tool}

Version one is project management software designed to support agile development methodologies such as Scrum. It is used by teams to ease in planning and managing multiple teams. Version One tool manages backlog items, tasks and bugs. It helps to plan and tracks sprints. Version One tool streamlines sprint reviews, generates burn down charts, conducts retrospectives and 


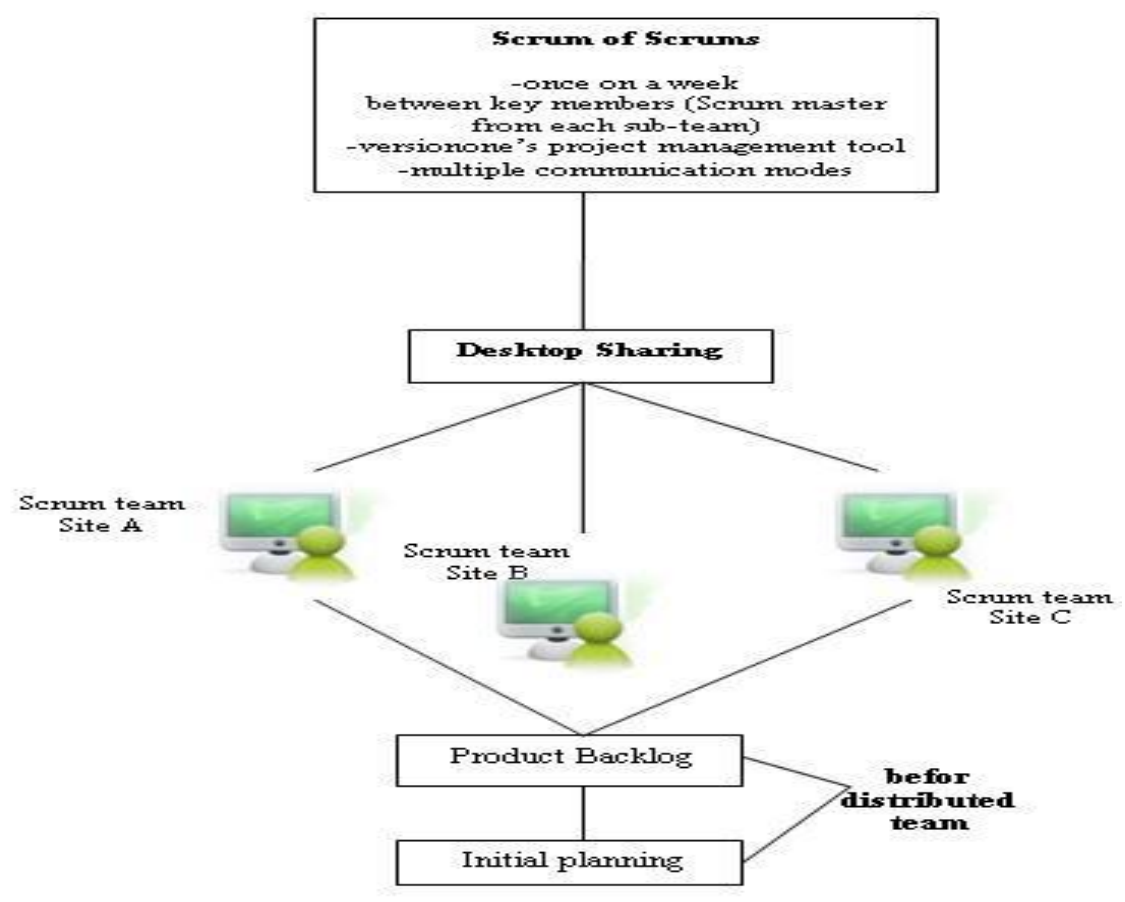

Fig 1. The Proposed Solution to Mitigate Communication Challenges in GSD

prioritizes product backlog and monitors scrum team impediments.

Stakeholders of Scrum trams are recommended to group at a single location before they are distributed. Team members develop first sprint as collocated teams. Distributed teams must agree on common definitions for Scrum terminologies, concepts, responsibilities and roles. Each team on each site in GSD is recommended to be autonomous. Scrum master of each team is instructed to coordinate and communicate. The proposed mitigation strategy is shown in fig. 1 .

The communication between teams in distributed sites is improved by developing a web application (Distributed Scrum Web Application) that helps teams to facilitate their work. Web application will provide different advantages as follows.

Security- It is ensured by specifying the privileges for each member by creating user account and assigning privileges to each one.

Availability- The web is available all the time and any member can access it.

Economical- Reducing costs on client side by requiring only the web browser that can access the server.

Web application helps teams by providing many services as following.

Organization- Each member in a team can access one place (website) to find what he wants to complete the work effectively. So, instead of using traditional tools like Skype or wiki the needed tools for communication or Scrum are built to provide security and reliability. This web application organizes the work between sites and within each site by scheduling all the work and meetings. Therefore, the web application provides reminder service about various activities.
Online Training- provides workshops that are presented by onshore site for distributed teams instead of visiting the onshore site to train. Moreover, workshops can be recorded and added to the library that contains recordings of either workshops or meetings that occurred. Hence, the time and cost of travelling are saved.

Suggestions- Each member can suggest and these suggestions can be discussed in suggestions meeting.

\section{A. Discussion}

Scrum development strictly follows tight deadlines. During planning phase, Scrum team requires good coordination among themselves and with product owner. Because at this stage team passing through understanding scope of project while requirements are gathering. Therefore it is necessary for product owner that he clearly understands scope of project and maintains good coordination with development team. This helps to improve team performance with successful delivery of sprint zero. To deliver Sprint zero during planning phase helps to engage customers by increasing their trust on methodology. Sprint zero reduces project risk and increase team moral to work with Scrum model.

Scrum team with low coordination results to increase work pressure and low quality product. Because team focus on to meat timelines of changing requirement environment of Scrum model. At this stage, team members require to act intelligently to cope with changing requirements and critical timelines. Implicit coordination and collaboration helps to improve team performance as a whole to achieve common goals and meet customer demands with maximum quality product. Through implicit coordination development team utilize their skills in more manageable way to beat work pressure and critical timelines fear. 


\section{EVALUATION OF THE PROPOSED SOLUTION}

Validation of the proposed solution is conducted through an electronic survey that is composed of nineteen questions. Questions are divided into three goals:

Goal 1- Geographical distance coordination and communication challenges are solved by the proposed mitigation strategies.

Goal 2- Scrum practices are effective to handle geographical coordination and communication challenges of distributed teams.

Goal 3- The effectiveness of the proposed solution to mitigate geographical distance communication challenges.

The data of survey is analyzed using SPSS software package and frequency tables and bar charts are used to display the results.

\section{A. Cumulative Statistical Analysis of Goal 1.}

The result of the analysis of the goal 1 is shown in Table 2. Table 2 shows that $60.04 \%$ of the respondents are agreed that geographical distance coordination and communication challenges are solved by the proposed mitigation strategies while $23.01 \%$ are strongly agreed to it. $16.95 \%$ of the respondents are remained neutral as shown in fig. 2 .

Table 2. Cumulative Analysis of Goal 1

\begin{tabular}{|c|c|c|c|c|c|}
\hline $\begin{array}{c}\text { Q. } \\
\text { No }\end{array}$ & $\begin{array}{c}\text { Strongly } \\
\text { Disagreed }\end{array}$ & Disagreed & Neutral & Agreed & $\begin{array}{c}\text { Strongly } \\
\text { Agreed }\end{array}$ \\
\hline 1 & 0.00 & 0.00 & 17.5 & 52.5 & 30 \\
\hline 2 & 0.00 & 0.00 & 25 & 55 & 20 \\
\hline 3 & 0.00 & 0.00 & 22.2 & 62.5 & 15 \\
\hline 4 & 0.00 & 0.00 & 7.5 & 65 & 27.5 \\
\hline 5 & 0.00 & 0.00 & 12.5 & 65 & 22.5 \\
\hline Total & 0.00 & 0.00 & 84.7 & 300 & 115 \\
\hline Avg. & 0.00 & 0.00 & 16.95 & 60.04 & 23.01 \\
\hline
\end{tabular}

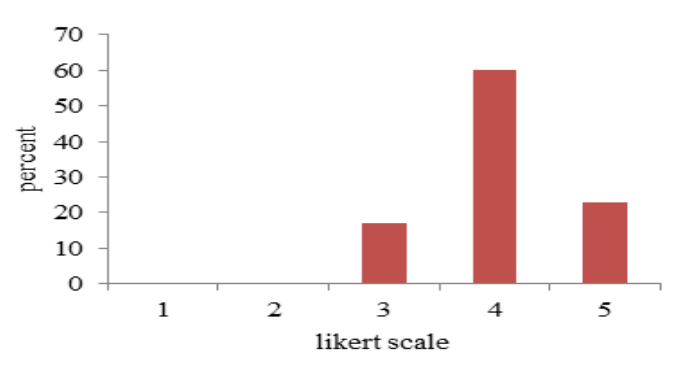

Fig 2. Graphical representation of goal 1

\section{B. Cumulative Statistical Analysis of Goal 2}

The result of the analysis of the goal 2 is shown in Table 3. Table 3 shows that that $61.25 \%$ of the respondents are agreed that Scrum practices are effective to solve the geographical coordination and communication challenges of distributed teams and $16.25 \%$ are strongly agreed to it. Whereas, $9.69 \%$ of the respondents are disagreed and $1.88 \%$ are strongly disagreed. $12.81 \%$ are remained neutral as shown below in fig. 3 .

Table 3. Cumulative Analysis of Goal 2

\begin{tabular}{|c|c|c|c|c|c|}
\hline $\begin{array}{c}\text { Q. } \\
\text { No }\end{array}$ & $\begin{array}{c}\text { Strongly } \\
\text { Disagreed }\end{array}$ & Disagreed & Neutral & Agreed & $\begin{array}{c}\text { Strongly } \\
\text { Agreed }\end{array}$ \\
\hline 6 & 15 & 40 & 10 & 25 & 10 \\
\hline 7 & 0.00 & 2.5 & 12.5 & 67.5 & 17.5 \\
\hline 8 & 0.00 & 7.5 & 10 & 65 & 17.5 \\
\hline 9 & 0.00 & 5 & 10 & 70 & 15 \\
\hline 10 & 0.00 & 2.5 & 15 & 62.5 & 20 \\
\hline 11 & 0.00 & 2.5 & 15 & 57.5 & 25 \\
\hline 12 & 0.00 & 2.5 & 12.5 & 75 & 10 \\
\hline 13 & 0.00 & 0.00 & 17.5 & 67.5 & 15 \\
\hline Total & 15 & 62.5 & 102.5 & 490 & 130 \\
\hline Avg. & 1.88 & 7.81 & 12.81 & 61.25 & 16.25 \\
\hline
\end{tabular}

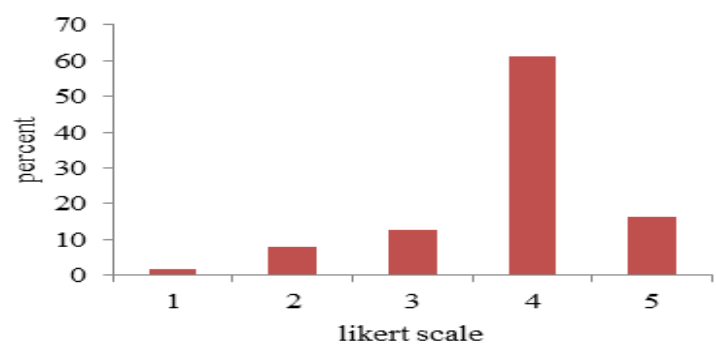

Fig 3. Graphical representation of goal 2

\section{Cumulative Statistical Analysis of Goal 3}

The result of the analysis of the goal 3 is shown in Table 4. Table 4 shows that $65.42 \%$ of the respondents are agreed and $21.25 \%$ are strongly agreed. $1.25 \%$ of the respondents are disagreed and $12.08 \%$ are remained neutral as shown in fig. 4 .

Table 4. Cumulative Analysis of Goal 3

\begin{tabular}{|c|c|c|c|c|c|}
\hline $\begin{array}{c}\text { Q. } \\
\text { No }\end{array}$ & $\begin{array}{c}\text { Strongly } \\
\text { Disagreed }\end{array}$ & Disagreed & Neutral & Agreed & $\begin{array}{c}\text { Strongly } \\
\text { Agreed }\end{array}$ \\
\hline 14 & 0.00 & 0.00 & 17.5 & 50 & 32.5 \\
\hline 15 & 0.00 & 2.5 & 15 & 67.5 & 15 \\
\hline 16 & 0.00 & 5 & 12.5 & 65 & 17.5 \\
\hline 17 & 0.00 & 0.00 & 7.5 & 77.5 & 15 \\
\hline 18 & 0.00 & 0.00 & 7.5 & 75 & 17.5 \\
\hline 19 & 0.00 & 0.00 & 12.5 & 57.5 & 30 \\
\hline Total & 0.00 & 7.5 & 72.5 & 392.5 & 127.5 \\
\hline Avg. & 0.00 & 1.25 & 12.08 & 65.42 & 21.25 \\
\hline
\end{tabular}

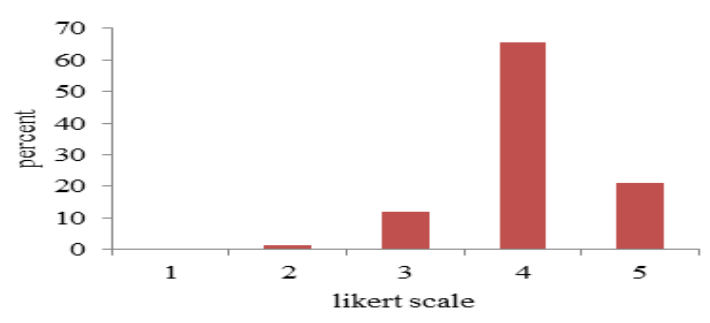

Fig 4. Graphical representation of goal 3 


\section{Final Cumulative Analysis of Three Goals}

The final cumulative analyses of three goals are shown in Table 5 and fig. 5. 62.24\% of the respondents are agreed with the proposed solution while $19.61 \%$ of the respondents are strongly agreed. $0.78 \%$ of the respondents are strongly disagreed whereas $3.68 \%$ are disagreed. However, $13.67 \%$ of the respondents are remained neutral.

Table 5. Cumulative Analysis of Three Goals

\begin{tabular}{|l|l|l|l|l|l|}
\hline $\begin{array}{l}\text { Goal. } \\
\text { No }\end{array}$ & $\begin{array}{l}\text { Strongly } \\
\text { Disagreed }\end{array}$ & Disagreed & Neutral & Agreed & $\begin{array}{l}\text { Strongly } \\
\text { Agreed }\end{array}$ \\
\hline 1 & & & 17.5 & 52.5 & 30 \\
\hline 2 & & & 25 & 55 & 20 \\
\hline 3 & & & 22.2 & 62.5 & 15 \\
\hline 4 & & & 7.5 & 65 & 27.5 \\
\hline 5 & & & 12.5 & 65 & 22.5 \\
\hline 6 & 15 & 40 & 10 & 25 & 10 \\
\hline 7 & & 2.5 & 12.5 & 67.5 & 17.5 \\
\hline 8 & & 7.5 & 10 & 65 & 17.5 \\
\hline 9 & & 5 & 10 & 70 & 15 \\
\hline 10 & & 2.5 & 15 & 62.5 & 20 \\
\hline 11 & & 2.5 & 15 & 57.5 & 25 \\
\hline 12 & & 2.5 & 12.5 & 75 & 10 \\
\hline 13 & & & 17.5 & 67.5 & 15 \\
\hline 14 & & & 17.5 & 50 & 32.5 \\
\hline 15 & & 2.5 & 15 & 67.5 & 15 \\
\hline 16 & & 5 & 12.5 & 65 & 17.5 \\
\hline 17 & & & 7.5 & 77.5 & 15 \\
\hline 18 & & & 7.5 & 75 & 17.5 \\
\hline 19 & & & 12.5 & 57.5 & 30 \\
\hline Total & 15 & 70 & 259.7 & 1182.5 & 372.5 \\
\hline Avg. & 0.78 & 3.68 & 13.67 & 62.24 & 19.61 \\
\hline
\end{tabular}

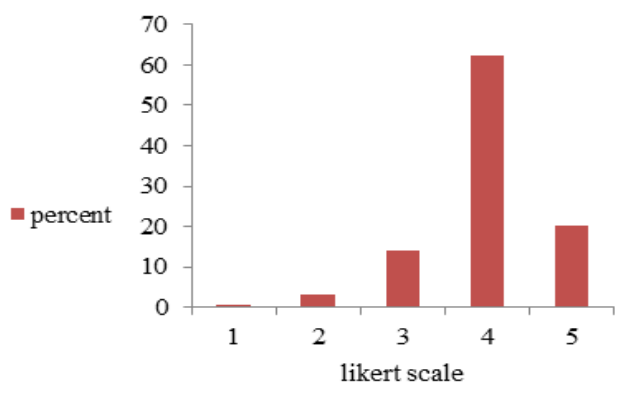

Fig 5. Graphical representation of cumulative 3 goals

\section{CONCLUSION AND FUTURE WORK}

Global software development (GSD) teams are located at different geographical places. GSD brings into several challenges to coordinate and communicate effectively among distributed teams. Many software development companies face the problem of coordination and communication in GSD projects. This paper proposes a novel solution to mitigate the coordination and communication challenges of GSD projects. A survey is conducted to validate the proposed solution. We conclude based on the results of survey that Scrum practices have a profound effect on GSD projects and Scrum model is beneficial to distributed development projects to mitigategeographical distance based communication and coordination challenges. Future work is to empirically validate the proposed solution using case study method.

\section{REFERENCES}

[1] Ramanujam, R. and Lee, I., "Collaborative and Competitive Strategies for Agile Scrum Development," Networked Computing and Advanced Information Management (NCM), 7th International Conference, 2011.

[2] Vlaanderen, K., Jansen, S., Brinkkemper, S., Jaspers, E., "The agile requirements refinery Applying SCRUM principles to software." Information and Software Technology, vol. 53, pp. 58-70, 2011.

[3] Alnuem, M., Ahmad, A., Khan, H., "Requirements Understanding: A Challenge in Global Software Development", IEEE 36th International Conference on Computer Software and Applications, 2012.

[4] Shah, Y., Raza, M., UlHaq, S., "Communication Issues in GSD" International Journal of Advanced Science and Technology, vol. 40, March, 2012.

[5] Sriram, R. and Mathew, S., "Global Software Development using Agile Methodologies: A Review of Literature", Proceeding of the IEEE ICMIT, 2012.

[6] Hossain, E., Babar, M., Paik, H., Verner, J., "Risk Identification and Mitigation Processes for Using Scrum in Global Software Development: A Conceptual Framework", 16th Asia-Pacific Software Engineering Conference, 2009.

[7] Scharff, C., Heng, S., Kulkarni, V., "On the Difficulties for Students to Adhere to Scrum on Global Software Development Projects: Preliminary Results", IEEE CTGDSD Conference, Zurich, 2012.

[8] Scharff, C., "Guiding Global Software Development Projects using Scrum and Agile with Quality Assurance", IEEE CSEE\&T Conference, New York: Honolulu/ HI, 2011.

[9] Su, H., "Some Management Principles Learned from Scrum Practices within a Global Software Development Project" International Journal of e-Education, e-Business, e-Management and e-Learning, vol. 1, no. 5, December. 2011.

[10] Gomes, V., Marczak, S., "Problems? We All Know We Have Them Do We Have Solutions Too?" IEEE Seventh International Conference on Global Software Engineering, 2012.

[11] Nurdiani, I., Jabangwe, R., Smitey, D., Damian, D., "Risk Identification and Risk Mitigation Instruments for Global Software Development: Systematic Review and Survey Results", Sixth IEEE International Conference on Global Software Engineering Workshops, 2011.

[12] Noordeloos, R., Manteli, C., Vliet, H., "From RUP to Scrum in Global Software Development: A Case Study," IEEE Seventh International Conference on Global Software Engineering, 2012.

[13] Hossain, E., Bannerman, P., and D. Jeffery, R., "Scrum Practices in Global Software Development: A Research 
Framework", 12th international conference, SpringerVerlag Berlin, Heidelberg, pp.88-102, 2011.

[14] Bannerman, P., Hossain, E., Jeffery, R., "Scrum Practice Mitigation of Global Software Development Coordination Challenges: A Distinctive Advantage" 45th Hawaii International Conference on System Sciences, 2012.

[15] Qureshi, M.R.J. and Alsulami, N. "Mitigating Coordination Costs in Global Software Development

[16] Cristal, M., Wildt, D. and Prikladnicki, R. Usage of SCRUM Practices within a Global Company. In: Engineering and Electronic Business, online June 2014; Using Scrum," International Journal of Information vol. 6, no. 3, 2014, pp. 16-21. Proceedings of the IEEE International Conference on Global Software Engineering, 2008, pp. 222-226.

[17] Hossain, E., Babar, M.A., and Paik, H. Using Scrum in Global Software Development: A Systematic Literature Review. In: Proceedings of the 4th IEEE International Conference on Global Software Engineering, 2009, pp. 175-184.

[18] Hossain, E., Bannerman, P. and Jeffery, D. Scrum Practices in Global Development: A Research Framework. In: Proceedings of the 12th International Conference on Product Focused Software Process Improvement (PROFES), 2011, pp. 88-102.

[19] Bannerman, P. L., Hossain, E. and Jeffery, R. Scrum Practice Mitigation of Global Software Development
Coordination Challenges: A Distinctive Advantage?" In: Proceedings of the 45th Hawaii International Conference on System Sciences, 2012, pp. 5309-5318.

[20] Nuevo, E.D., Piattini, M., and Pino, F.J. Scrum-based Methodology for Distributed Software Development. In: Proceedings of the 6th IEEE International Conference on Global Software Engineering, 2011, pp. 66-74.

\section{Authors' Profiles}

Areej AL_Zaidi: Post-graduate student for master degree for Information Technology in King Abdul-Aziz University.

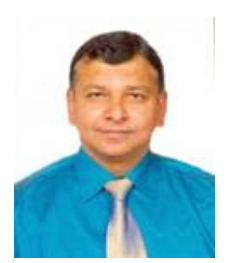

M. Rizwan Jameel Qureshi: Associate Professor of Information Technology, Faculty of Computing \& Information Technology, King Abdul-Aziz University, major in software engineering and database management systems.

How to cite this paper: Areej Sewalh AL_Zaidi, M. Rizwan Jameel Qureshi,"Scrum Practices and Global Software Development", IJIEEB, vol.6, no.5, pp.22-28, 2014. DOI: 10.5815/ijieeb.2014.05.04 